\title{
Antenatal Cocaine Exposure Produces Accelerated Surfactant Maturation without Stimulation of Antioxidant Enzyme Development in the Late Gestation Rat
}

\author{
ILENE R. S. SOSENKO \\ Divisions of Neonatology and Pulmonary Research, Departments of Pediatrics and Internal Medicine. University \\ of Miami School of Medicine. Miami. Florida 33101
}

\begin{abstract}
Antenatal cocaine administration is associated with increases in catecholamines and ghicocorticoids and results in fetal hypoxemia, with the speculated potential for increased free radical formation in the fetus. Because of this, we hypothesized that antenatal cocaine exposure would produce not only accelerated pulmonary surfactant maturation, but also stimulation of antioxidant enzyme (AOE) system maturation and the ability to mount a protective AOE response to hyperoxia as well. Seventytwo, 48, and $24 \mathrm{~h}$ before delivery at either 20,21 , or $22 \mathrm{~d}$ of gestation, pregnant rats received twice daily s.c. injections of cocaine hydrochloride $(40 \mathrm{mg} / \mathrm{kg})$ or saline. Offspring demonstrated growth retardation with significant reduction in body weight at all 3 gestational $d$ studied that persisted to the 5th postnatal d. Serum corticosterone and total catecholamines were elevated from 50 to $200 \%$ in cocaine-exposed adult rats and pups. Advanced surfactant development in cocaine-exposed fetal offspring was apparent at $d 21$ of gestation, with significant increases in lung disaturated phosphatidylcholine content in cocaine $(3.94 \pm$ $0.67 \mathrm{mg} / \mathrm{g}$ lung) versus control $(3.25 \pm 0.64)$ offspring $(p$ $<0.01)$. No increases in AOE maturation were found in cocaine-exposed fetuses at all 3 gestational d studied, whereas a significant decrease was demonstrated in glutathione peroxidase activity at $\mathrm{d} 20$ and 22 of gestation in cocaine-exposed fetuses. In addition, cocaine newborns manifested a protective $\mathrm{AOE}$ response to hyperoxia of a magnitude similar to that of control newborns. These findings suggest that cocaine may be affecting $\mathrm{AOE}$ system maturation through hormones other than glucocorticoids and catecholamines and indicate that the pulmonary surfactant and AOE systems may share some, but not all, of the same influences on their late gestational development. (Pediatr Res 33: 327-331, 1993)
\end{abstract}

\section{Abbreviations}

AOE, antioxidant enzyme

SOD, superoxide dismutase

CAT, catalase

GP, glutathione peroxidase

DSPC, disaturated phosphatidylcholine
In comparison to other commonly abused substances in pregnancy, gestational cocainc abusc of socictal magnitude is a relatively recent phenomenon. For this reason, questions as to cocaine's effects on various aspects of fetal development and physiology are in the process of being actively investigated. In terms of its effects on the development of the lung, antenatal cocaine exposure has been associated with enhanced fetal surfactant maturation (1); however, its effects on AOE development remain largely unexplored.

Cocaine has been demonstrated to produce both hormonal and physiologic effects on the developing fetus that could potentially impact on the maturation of the AOE system. First, in addition to its major neurochemical effect of blockade of the reuptake of catecholamines (2), cocaine is presumed to stimulate endogenous glucocorticoids (3). Second, cocaine administered to pregnant ewes has been found to decrease uterine blood flow and produce marked fetal hypoxemia (4) and is speculated to potentially increase free radical formation in the fetus.

Previous studies have demonstrated that both exogenous and endogenous glucocorticoid hormones stimulate AOE maturation as well as surfactant maturation in the rat $(5,6)$. Specifically, dexamethasone administered to pregnant rats in late gestation produced accelerations in surfactant and AOE development of similar magnitude (5). In addition, blockade of endogenous glucocorticoid production using metyrapone resulted in a delay in both surfactant and AOE maturation (6). In terms of the influence of hypoxia on the AOE, studies in adult rats exposed to hypoxia have demonstrated increases in the AOE protective response, speculated to be related to increased production of oxygen free radicals under hypoxic conditions (7). When adult animals (rats, mice, and rabbits) were compared with neonatal animals in terms of their response to hyperoxia ( $>95 \%$ oxygen), the adult animals demonstrated a survival time of 3 to $5 \mathrm{~d}$ without significant $\mathrm{AOE}$ elevations during that time period, whereas the neonatal animals survived $>7 \mathrm{~d}$ and demonstrated significant AOE elevations by $24 \mathrm{~h}$ of hyperoxic exposure.

Thus, because of evidence of increased glucocorticoid stimulation with cocaine coupled with the accelerating role that glucocorticoid hormones have been shown to play on AOE maturation, and because the alteration of fetal oxygenation with potential increased free radical formation by cocaine would suggest an additional stimulatory role for cocaine on the development of the AOE, we hypothesized that offspring of rats exposed to cocaine would manifest stimulation not only of surfactant development but of AOE development and of a greater protective $\mathrm{AOE}$ response as well.

\section{MATERIALS AND METHODS}

Correspondence: Ilene R. S. Sosenko, M.D. Pulmonary Research (R-120). University of Miami School of Medicine, P.O. Box 016960. Miami, FL 33101 Supported in part by American Lung Association of Florida Career Investigator Award.
Animals and treatments. Using adult Sprague-Dawley albino female rats $(\sim 250 \mathrm{~g})$, timed pregnancies were achieved by placing 
male and female rats together overnight, checking for spermpositive vaginal smears the following morning, and using the midpoint of the cohabitation period as the onset of pregnancy. The timed-pregnant rats were maintained on standard laboratory food and water ad libitum and kept on a 12-h:12-h light-dark cycle.

The animal protocol was preapproved by the University of Miami Research Animal Welfare Committee.

At 72,48 , and $24 \mathrm{~h}$ before premature delivery at 20 or $21 \mathrm{~d}$ or term delivery at $22 \mathrm{~d}$ gestation, pregnant rats were injected s.c. twice daily with cocaine hydrochloride (Sigma Chemical Co., St. Louis, MO), $40 \mathrm{mg} / \mathrm{kg} /$ dose. Control rats received equivolumes of saline according to the same schedule.

For the developmental studies, $72 \mathrm{~h}$ after the first injection, rat fetuses of 20 or 21 d gestation were delivered by hysterotomy under sodium pentobarbital anesthesia. Full-term newborn rats (22 d) were obtained either by hysterotomy or after normal parturition. After weight of the pups was recorded, lungs were prepared for biochemical determinations as described below.

Oxygen exposures. To study the hyperoxic response, separate litters of cocaine and control rats were allowed to deliver spontaneously and were then placed in either $>95 \%$ oxygen or room air for $5 \mathrm{~d}$ as previously described (8). Several newly delivered litters from cocaine-injected dams were pooled and then randomly distributed to "surrogate," noncocaine-injected dams that had recently delivered for hyperoxia or air exposure. Similarly, several newly delivered control litters were pooled and then randomly distributed to dams that had recently delivered. Body weights of air-exposed cocaine and control offspring during the first 5 postnatal d were obtained as a measure of postnatal growth of the two groups. After exposure, lungs were examined for AOE response to exposure as described below.

Hormonal analysis. From random dams and litters, as well as female rats receiving the appropriate protocol of saline or cocaine injections and subsequently found not to be pregnant, blood was collected, and serum corticosterone and total catecholamines were assayed using specific RIA test kits: 1) corticosterone (Inter Sci Diagnostics, Inglewood, CA) with a sensitivity of $20 \mathrm{pg}$ and a cross-reactivity of $100 \%$ corticosterone, $28 \%$ cortisol, and $3.6 \%$ cortisone; 2) total catecholamines (Amersham, Arlington Heights, IL) with a sensitivity from 2 to $5 \mathrm{pg}$ for norepinephrine and epinephrine and 15 to $20 \mathrm{pg}$ for dopamine per $50-\mu \mathrm{L}$ sample and an average coefficient of variation of approximately $4 \%$. In general, for fetal specimens, pooling of serum from an entire litter was required to obtain an adequate quantity for the assays.

Lung biochemistry. Fetal or newborn lungs were perfused immediately in situ via the pulmonary artery using cold saline after the left atrial appendage had been resected to allow drainage of the perfusate. The perfused lungs were then removed, stripped of nonpulmonary tissue, and blotted dry. Lungs that appeared to have been poorly perfused in terms of blood retention or retention of perfusate were discarded. Postperfused wet lung weight was obtained. Random unperfused newborn lungs were also weighed and then dried at $80^{\circ} \mathrm{C}$ for $72 \mathrm{~h}$ for wet-weight:dryweight ratio calculations. The perfused lungs were homogenized in 20 to 30 times their weight of cold saline in a Brinkman polytron (high speed $\sim 90 \mathrm{~s}$ ). For preterm or small newborns, two to four lungs were pooled per sample to obtain adequate tissue for the assays. Samples were not specifically chosen to separate male and female fetuses.

Aliquots of the homogenate were subsequently assayed for AOE activities using standard spectrophotometric assays for total SOD (9), CAT (10), GP (11), DNA (12), and protein (13). Purified enzyme standards (SOD and CAT) and DNA standard were obtained from Sigma Chemical and GP was obtained from Boehringer Mannheim (Indianapolis, IN). Results of AOE analyses were expressed as units of enzyme activity per mg of DNA.

For phospholipid analysis, a portion of lung homogenate was first lipid-extracted using the method of Bligh and Dyer (14) and then assayed for total lipid phosphorus following the method of
Morrison (15). For DSPC analysis, a separate aliquot of lipid extract was reacted with osmium tetroxide as described by Mason et al. (16) and subsequently assayed for lipid phosphorus (15). A known quantity of ${ }^{14} \mathrm{C}$-dipalmitoylphosphatidylcholine (New England Nuclear, Boston, MA) was added before lipid extraction to provide estimation of and correction for sequential losses. Total phospholipid and DSPC content were expressed as $\mathrm{mg} / \mathrm{g}$ postperfused wet lung weight, and DSPC was also calculated per mg protein and per mg DNA.

Statistics. Statistics were determined using unpaired $t$ test. When multiple $t$ tests were performed (i.e. AOE data, physical measurements), a Bonferroni-type correction was made, allowing as significant a $p$ value of less than 0.01 (17).

\section{RESULTS}

Despite an increase in mortality of cocaine-injected pregnant dams (approximately 10 to $15 \%$ ), no significant differences were found between cocaine and control litters in terms of in utero fetal demise or obvious congenital malformations. However, after spontaneous delivery at $22 \mathrm{~d}$ (term), cocaine-exposed dams had a markedly greater likelihood of harming and/or ignoring the newly born pups.

The physical measurements of postperfused wet lung weight, body weight, and lung-weight:body-weight ratios of cocaine and control offspring of 20 to $22 \mathrm{~d}$ of gestation are found in Table 1. Cocaine offspring at all three gestational ages demonstrated a significant reduction in body weight compared with controls; lung weight was significantly decreased in 21 -d cocaine offspring $(p<0.01)$. The reduction in lung weight in 20-d cocaine offspring reached a $p$ value of $<0.05$ (not considered to be significant according to our statistical correction for multiple $t$ tests). Lungweight:body-weight ratios were not different between groups at the 3 gestational $d$ studied. In addition, no differences were present in lung DNA or protein content (mg/g lung) in cocaine versus control offspring at any gestational day examined (data not shown) or in wet-lung-weight:dry-lung-weight ratios $(5.85 \pm$ 0.20 versus $5.73 \pm 0.12$ ) for newborn cocaine versus control offspring, respectively.

Figure 1 extends measurements of body weight from the day of birth to the 5 th postnatal d in cocaine offspring (nurtured by control, surrogate dams) and control offspring. Cocaine offspring were found to have postnatal growth that was parallel to but did not "catch up" to the early postnatal growth of the control pups. Body weight of 5-d-old cocaine pups was significantly reduced $(p<0.01)$ compared with the weight of 5-d-old control pups.

Serum corticosterone and total catecholamine levels in cocaine-injected and control rats and offspring are found in Table 2. Cocaine-injected rats demonstrated a $50 \%$ increase in corticosterone and a significant $200 \%$ increase in total catecholamines compared with controls. Increases in cocaine offspring were of similar magnitude with an increase in corticosterone of $125 \%$

Table 1. Physical measurements of cocaine and control rat offspring*

BW LW LW/BW

$\begin{array}{cccc}20 \mathrm{~d} & & & \\ \text { Cocaine } & 2.45 \pm 0.28 \dagger & 0.1084 \pm 0.011 & 0.0313 \pm 0.002 \\ \text { Control } & 3.82 \pm 0.23 & 0.1299 \pm 0.015 & 0.0328 \pm 0.003 \\ 21 \mathrm{~d} & & & \\ \text { Cocaine } & 4.24 \pm 0.59 \dagger & 0.1127 \pm 0.016 \dagger & 0.0269 \pm 0.003 \\ \quad \text { Control } & 5.42 \pm 0.28 & 0.1476 \pm 0.023 & 0.0272 \pm 0.004 \\ 22 \mathrm{~d} & & & \\ \text { Cocaine } & 5.44 \pm 0.63 \dagger & 0.1029 \pm 0.012 & 0.0191 \pm 0.003 \\ \text { Control } & 6.46 \pm 0.65 & 0.1164 \pm 0.202 & 0.0198 \pm 0.003\end{array}$

${ }^{*} n=3$ to 6 litters/group/gestational age. BW, body weight; LW, postperfused, wet lung weight. Weight units in $\mathrm{g}$.

$\dagger p<0.01$. 


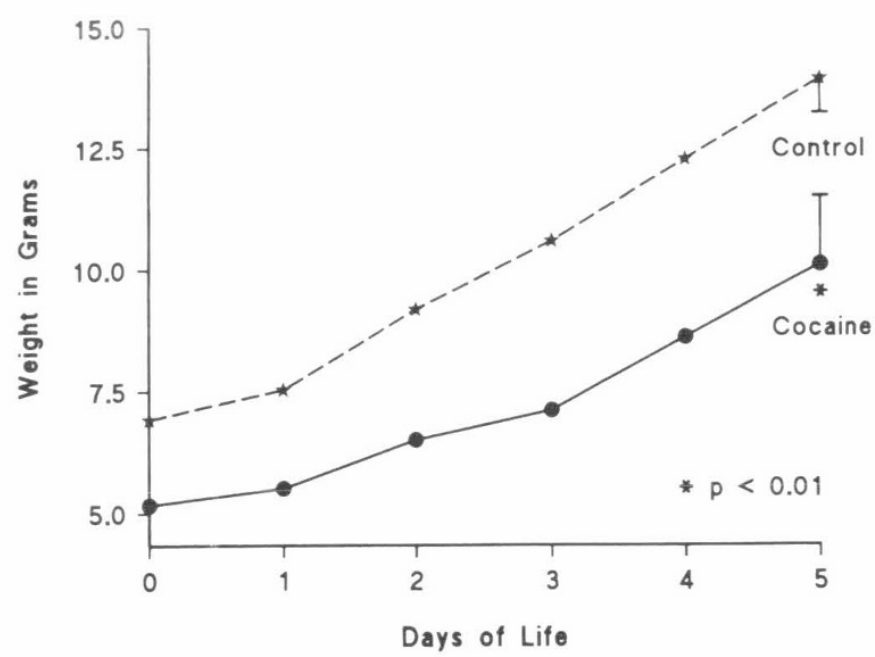

Fig. 1. Postnatal growth of cocaine and control offspring during the first 5 postnatal d. Each group represents the pooling of two litters to obtain the average weight of 8 to 10 pups/group. Days of Life on the $x$ axis represents postnatal days. On the Sth postnatal d, weights of individual pups were obtained; therefore, means, SD, and statistics could be calculated at that time. Differences were significant at the $p<0.01$ level.

and in total catecholamine levels of $149 \%$ compared with control offspring.

AOE and surfactant development in 20- to 22-d cocaine versus control offspring are outlined in Table 3. Similar results for surfactant development were also obtained when DSPC for cocaine versus control, respectively, was expressed per mg protein (gestational d 20: $0.0293 \pm 0.0049$ versus $0.0275 \pm 0.0028$; gestational d 21: $0.0753 \pm 0.0276$ versus $0.0500 \pm 0.0091, p<$ 0.01 ; gestational d 22-hysterotomy: $0.0365 \pm 0.0081$ versus $0.0349 \pm 0.0035$; gestational d 22-spontaneous birth: $0.0699 \pm$ 0.0055 versus $0.0733 \pm 0.0129$ ) and per mg DNA (gestational d 20: $0.1275 \pm 0.0204$ versus $0.1196 \pm 0.0160$; gestational d 21: $0.4148 \pm 0.0712$ versus $0.3508 \pm 0.0074, p<0.05$; gestational d 22-hysterotomy: $0.1959 \pm 0.0409$ versus $0.1969 \pm 0.0058$; gestational d 22 -spontaneous birth: $0.2503 \pm 0.0280$ versus 0.2633 $\pm 0.3191)$. Whereas 21 -d cocaine offspring demonstrated advanced surfactant maturation with a significant increase in lung DSPC content, no acceleration of AOE maturation was apparent, e.g. no increases in total SOD, CAT, or GP activities were present in cocaine versus control offspring at the 3 gestational d studied. Unexpectedly, cocaine offspring demonstrated significant decreases in GP activities at d 20 and 22 of gestation.

The ability to increase AOE activities after $5 \mathrm{~d}$ of $>95 \%$ oxygen in cocaine and control newborn rats is graphically represented in Figure 2. AOE activity levels were first calculated as activity units/mg DNA and then expressed as a percentage of change in oxygen-exposed lung activity levels compared with air-exposed lung values. Both cocaine and control newborn rats were able to induce a significant increase in AOE with hyperoxia, representing a protective $\mathrm{AOE}$ of similar magnitude to $5 \mathrm{~d}$ of hyperoxic exposure. No significant differences were present between the cocaine and control offspring in terms of DSPC response to $5 \mathrm{~d}$ of hyperoxia.

\section{DISCUSSION}

The choice of the cocaine dose for the present study, $40 \mathrm{mg} /$ $\mathrm{kg}$, was based on previous data that demonstrated the threshold for maternal mortality to be at 50 to $60 \mathrm{mg} / \mathrm{kg}$ (18). The s.c. route of administration was selected because reports have indicated that s.c. administration results in a combination of dermal necrosis and vasoconstriction, producing plasma levels of cocaine that peak slowly and result in prolonged tissue exposure to the drug $(19,20)$. As a result of our dosage and route of administration, offspring of cocaine-injected pregnant rats demonstrated evidence of growth retardation, with significant decreases in body weight present from $20 \mathrm{~d}$ of gestation to (at least) the 5 th postnatal d. These findings are in agreement with some (21) but not all previous studies (22). Although several investigators have reported that female offspring demonstrate increased sensitivity to the effects of antenatal cocaine (23), we did not determine fetal sex and thus did not examine differential sex effects.

In addition to growth retardation, cocaine exposure during pregnancy has been found to produce a wide array of deleterious influences on the developing offspring, ranging from teratogenesis (24) to abnormalities in respiratory control in both fetus (25) and newborn (26). In terms of maternal-fetal physiology, i.v. cocaine administered to pregnant ewes produced a doseresponse relationship with decreased placental blood flow and increased uterine vascular resistance, increased fetal heart rate, and decreased fetal oxygen content $(4,27)$. In relation to hormonal effects, cocaine has been found to block the reuptake of catecholamines at the nerve terminal, thus increasing circulating catecholamine levels (2). In human infants who had been exposed to cocaine in utero, norepinephrine levels were found to be elevated as late as 2 mo of age (28). Our findings in the present study of an almost doubling of total catecholamine levels in cocaine-exposed adult and newborn rats are consistent with these previous findings. Not only has cocaine been demonstrated to elevate catecholamines, but it has also been reported to increase corticosterone in rats by stimulation of the pituitary-adrenal axis with resultant ACTH release (3), findings similar to our 50 to $125 \%$ elevation in serum corticosterone levels in adult and newborn cocaine-exposed rats.

Despite the extensive evidence of the growth retarding, teratogenic, physiologic, and hormonal effects of prenatal cocaine

Table 2. Serum corticosterone and total catecholamine levels in cocaine-injected and control rats and pups*

\begin{tabular}{|c|c|c|c|}
\hline & Cocaine & Control & Change \\
\hline \multicolumn{4}{|l|}{ Corticosterone $(\mu \mathrm{g} / \mathrm{dL}) \dagger$} \\
\hline $\begin{array}{l}\text { Pregnant rats and rats that have recently given birth } \\
n\end{array}$ & $\begin{array}{c}0.990 \pm 0.37 \\
6\end{array}$ & $\begin{array}{c}0.660 \pm 0.14 \\
5\end{array}$ & $\uparrow 50 \%$ \\
\hline Pups & $0.540 \pm 0.24$ & 0.240 & $\uparrow 125 \%$ \\
\hline Litters & 3 & 1 & \\
\hline \multicolumn{4}{|l|}{ Total catecholamines $(\mathrm{pg} / \mathrm{mL}) \ddagger$} \\
\hline $\begin{array}{l}\text { Nonpregnant females } \\
n\end{array}$ & $\begin{array}{c}13606 \pm 7089 \S \\
7\end{array}$ & $\begin{array}{c}4542 \pm 6294 \\
6\end{array}$ & $\uparrow 200 \%$ \\
\hline Pups & $19381 \pm 15169$ & $7798 \pm 8498$ & $\uparrow 149 \%$ \\
\hline Litters & 3 & 5 & \\
\hline
\end{tabular}

* Hormonal assays were performed by specific RIA kits. Nonpregnant females were rats assessed to be pregnant postmating, receiving complete course of cocaine or saline injections and then found to be nonpregnant at term.

† SI unit conversion: $\mu \mathrm{g} / \mathrm{dL} \times 28.86=\mathrm{nmol} / \mathrm{L}$.

$\ddagger$ SI unit conversion: $\mathrm{pg} / \mathrm{mL} \times 5.677=\mathrm{pmol} / \mathrm{L} \div 1000=\mathrm{nmol} / \mathrm{L}$.

$\S p<0.05$. 
Table 3. AOE and surfactant development of cocaine and control rat offspring*

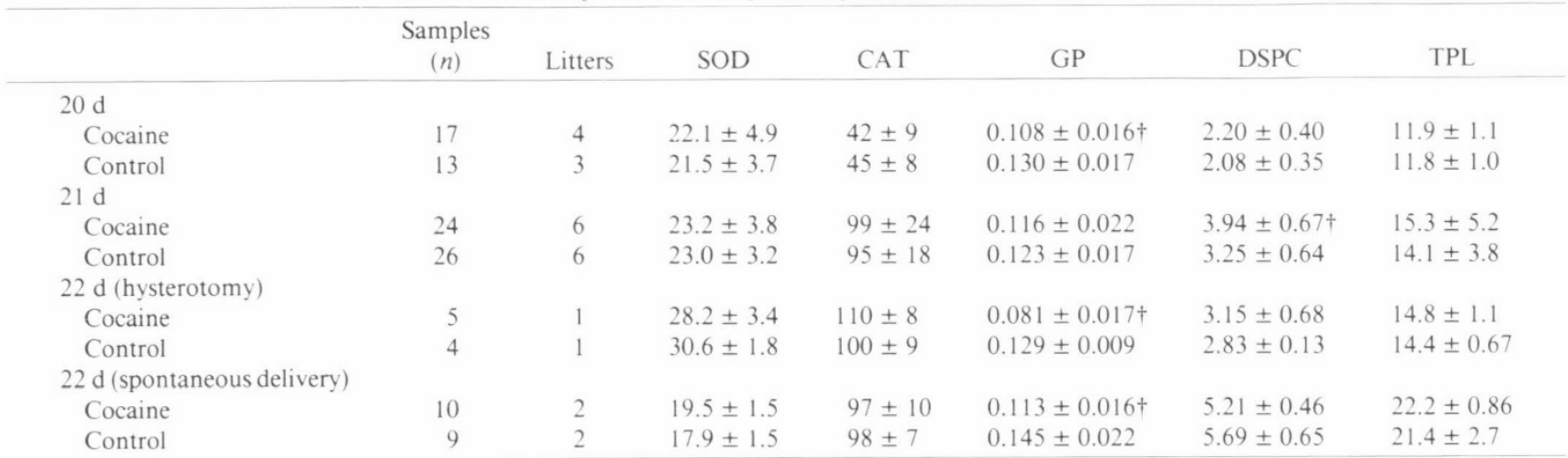

* Units for AOE $=\mathrm{IU} / \mathrm{mg}$ DNA. TPL, total phospholipid. DSPC and TPL are both expressed as mg/g postperfused wet lung weight. $+p<0.01$.

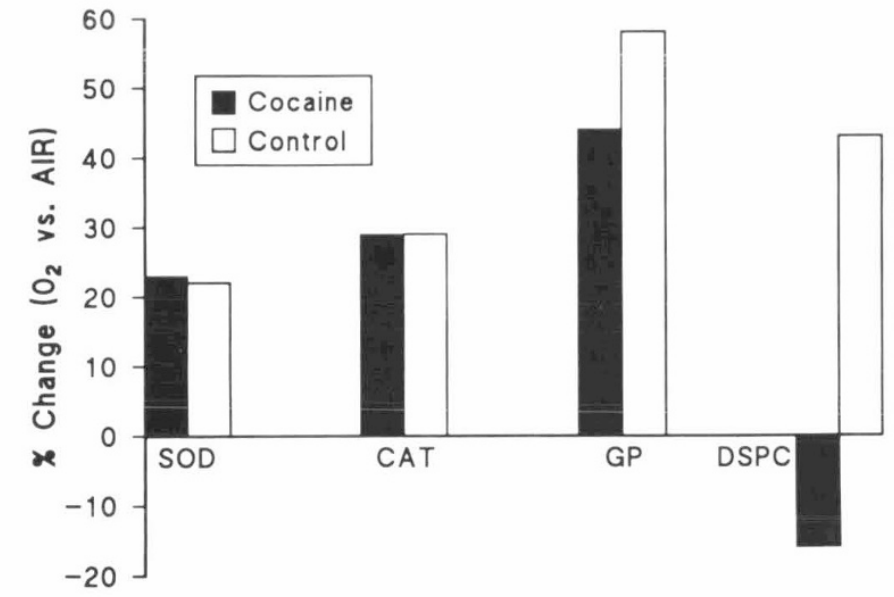

Fig. 2. AOE and DSPC response to $5 \mathrm{~d}$ of hyperoxia $\left(>95 \% \mathrm{O}_{2}\right)$ in cocaine-exposed and control newborn rats. Two litters from each group were pooled and divided into a hyperoxia group and an air group. Values are expressed as the percentage of difference between hyperoxia-exposed and air-exposed activity levels. Both groups demonstrated a significant increase $(p<0.05)$ in AOE activities when hyperoxia-exposure values were compared with air values. No difference in magnitude of response was present between cocaine and control offspring in terms of AOE or DSPC.

exposure, there remains a relative paucity of data in relation to cocaine's effect on fetal lung maturation, specifically the development of the surfactant and AOE systems. Epidemiologic investigations have failed to yield conclusive data as to whether antenatal cocaine exposure in human infants reduces the incidence of hyaline membrane disease $(29,30)$. In the rabbit, cocaine has been reported to alter fetal lung pressure-volume characteristics (with increased distensibility and retention of air) and increase bubble stability, characteristics of enhanced surfactant maturation, as well as accelerate morphologic lung maturation (1). Our biochemical findings of a significant increase in lung DSPC content at $21 \mathrm{~d}$ of gestation in the rat are consistent with these previously reported surfactant-related findings in the rabbit. The mechanism by which cocaine accelerates fetal lung maturation may relate to the previously reported as well as presently demonstrated increased catecholamines and/or glucocorticoids.

Glucocorticoids, both endogenous and exogenous, have been shown to play a significant role in AOE maturation as well as surfactant maturation $(5,6)$. In addition, in adult rats, increased free radical production related to hypoxia is associated with enhanced pulmonary AOE activities (7). Thus, because of the increased glucocorticoids associated with cocaine exposure and because of the reported fetal hypoxemia associated with maternal cocaine, we hypothesized that cocaine administration to pregnant rats would produce acceleration in fetal lung AOE maturation and enable the cocaine offspring to mount a heightened AOE response to hyperoxic exposure. Neither hypothesis was found to be true for the fetal and newborn offspring of cocainetreated pregnant rats in the present investigation. There are a number of possible explanations for our findings of lack of stimulation of AOE by antenatal cocaine. First, cocaine's stimulation of endogenous glucocorticoids may not have been of sufficient magnitude to influence AOE development. Second, in addition to its stimulation of glucocorticoids, cocaine may have produced increases in other hormones not examined here, such as the thyroid hormones [because catecholamines have been reported to stimulate thyroid hormone synthesis and release (31)]. Previous investigations have determined that, despite a stimulation of surfactant maturation, thyroid hormone fails to stimulate AOE maturation and actually results in a delay in AOE system development (32). This could be an explanation for the finding of decreased GP activity in cocaine versus control lung at gestational d 20 and 22. Finally, antenatal cocaine may be acting on both lung systems through mechanisms involving neither hormones nor increased free radical production. Nonetheless, the findings of the present study are yet another example of the fact that the surfactant system and AOE system share some, but not all, of the same mechanistic influences on their late gestational maturation in the developing lung.

Acknowledgments. The author thanks Miguel Martinez and Dr. Lalitha Price for their excellent technical support, Dr. Lee Frank for his scientific and emotional support, and Dr. Emmalee $\mathrm{S}$. Bandstra for her critical review of the manuscript.

\section{REFERENCES}

1. Kain ZN, Chinoy MR, Antonio-Santiago MT, Marchitelli RN, Scarpelli EM 1991 Enhanced lung maturation in cocaine-exposed rabbit fetuses. Pediatr Res 29.534-537

2. Muscholl E 1961 Effect of cocaine and related drugs on the reuptake of noradrenaline by heart and spleen. Br J Pharmacol Chemother 16:352-359

3. Moldow RL, Fischman AJ 1987 Cocaine induced secretion of ACTH, betaendorphine and corticosterone. Peptides 8:819-822

4. Woods JR, Plessinger MA, Clark KE 1987 Effect of cocaine on uterine blood flow and fetal oxygenation. JAMA 257:957-961

5. Frank L, Lewis PL, Sosenko IRS 1985 Dexamethasone stimulation of fetal rat lung antioxidant enzyme activity in parallel with surfactant stimulation. Pediatrics 75:569-574

6. Sosenko IRS, Lewis PL, Frank L 1986 Metyrapone delays surfactant and antioxidant enzyme maturation in developing rat lung. Pediatr Res 20:672675

7. Frank L 1982 Protection from pulmonary oxygen toxicity by preexposure of rats to hypoxia: role of the antioxidant enzyme system. J Appl Physiol 53:475-482

8. Frank L, Bucher JR, Roberts RJ 1978 Oxygen toxicity in neonatal and adult animals of various species. J Appl Physiol 45:699-704 
9. McCord JM, Fridovich I 1969 Superoxide dismutase: an enzymic function for erythrocuprein (hemocuprein). J Biol Chem 244:6049-6055

10. Holmes RS, Masters CJ 1970 Epigenetic interconversion of the multiple forms of mouse liver catalase. FEBS Lett 11:45-48

11. Paglia DE, Valentine WN 1967 Studies on the quantitative and qualitative characterization of erythrocyte glutathione peroxidase. J Lab Clin Med $70: 158-159$

12. Richards G 1974 Modifications of the diphenylamine reactions giving increased sensitivity and simplicity in the estimation of DNA. Anal Biochem 57:369376

13. Schacterle RE, Pollack RL 1973 A simplified method for the quantitative assay of small amounts of protein in biological material. Anal Biochem 51:654655

14. Bligh EF, Dyer WJ 1959 A rapid method of total lipid extraction and purification. Can J Biochem Physiol 37:911-917

15. Morrison WR 1964 A fast, simple and reliable method for the microdetermination of phosphorus in biological materials. Anal Biochem 11:218-224

16. Mason RJ, Nellenbogen J, Clements JA 1976 Isolation of disaturated phosphatidylcholine with osmium tetroxide. J Lipid Res 17:281-284

17. Fisher RA 1970 Statistical Methods for Research Workers. Hafner Press, New York, pp 213-224

18. Church MW, Dintcheff BA, Gessner PK 1988 Dose-dependent consequences of cocaine on pregnancy outcome in the Long-Evans rat. Neurotoxicol Teratol 10:51-58

19. Benuck M, Lajtha A, Reith MEA 1987 Pharmacokinetics of systemically administered cocaine and locomotor simulation in mice. J Pharmacol Exp Ther 243:144-149

20. Dow-Edwards DL 1989 Fetal and maternal plasma cocaine levels peak rapidly following cocaine administration in rat. Ann NY Acad Sci 562:280-289

21. Zuckerman B, Frank DA, Hingson R. Amaro H, Levenson SM, Kayne H,
Parker S, Vinci R, Aboagve K. Fried LE, Timperi A. Bauchner H 1989 Effects of maternal marijuana and cocaine use on fetal growth. $\mathrm{N}$ Engl J Med 320:762-768

22. Hutchings DE. Fico TA, Dow-Edwards DL 1989 Prenatal cocaine: maternal toxicity, fetal effects and motor activity in the offspring. Neurotoxicol Teratol 11:65-69

23. Hughes JE, Freed LA. Donohue LM. Dow-Edwards DL 1990 Gender differences in the effects of prenatal cocaine exposure on locomotor activity in 21-22 day old rats. Soc Neurosci Abstr 16:243

24. Mahalik MP, Gautieri RF, Mann DE 1984 Mechanisms of cocaine-induced teratogenesis. Res Commun Subst Abuse 5:279-302

25. Gingras J. Hume R. O’Donnell KE. Stranger C 1989 Atypical fetal breathing patterns associated with in utero cocaine exposure. Pediatr Res 25: $310 \mathrm{~A}(\mathrm{abstr})$

26. Chasnoff IJ, Hunt CE, Kletter R, Kaplan D 1989 Prenatal cocaine exposure is associated with respiratory pattern abnormalities. Am J Dis Child 143:583587

27. Moore TR, Sorg J, Miller L, Key TC. Resnik R 1986 Hemodynamic effects of intravenous cocaine on pregnant ewe and fetus. Am J Obstet Gynecol 155:883-888

28. Davidson Ward SL, Schuetz S, Wachsman L, Bean XD, Bautista D, Buckley S, Sehgal S. Warburton D 1991 Elevated plasma norepinephrine levels in infants of substance-abusing mothers. Am J Dis Child 145:44-48

29. Wu S, Raval D. Anyebuno M. Wieus A. Pildes RS 1989 Does cocaine affect pulmonary status of LBW infants? Pediatr Res 25:236A(abstr)

30. Maynard EC, Dreyer SA, Oh W 1989 Prenatal cocaine exposure and hyaline membrane disease (HMD). Pediatr Res 25:223A(abstr)

31. Landsberg L. Young JB 1987 Catecholamines and the sympathoadrenal sys-

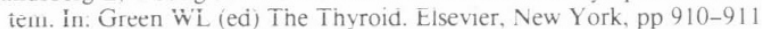

32. Sosenko IRS, Frank L 1987 Thyroid hormone depresses antioxidant enzyme maturation in fetal rat lung. Am J Physiol 253:R592-R598 